\title{
Modelo de la Corteza Continental en Tegucigalpa por medio del análisis de la Función Receptora
}

\author{
Luis VARGaS \\ Universidad Nacional Autónoma de Honduras \\ luisfisicaunah@gmail.com \\ Resumen
}

On this work its presented the study of all the recorded events for the seismological station TGUH located at Tegucigalpa, Honduras since september, 2006 to july of 2014. Those events were selected under certain criteria and specific characteristics that should be fulfilled for their use in the determination of the continental crust thickness under the station that recorded them, as the ration that exist between the propagation velocities $V_{p} / V_{s}$, based on the analysis of the Receiver Function method with the Stacking Method $(H-\kappa)$ the results from that are the estimated Bulk parameters were a thickness of 34.8 kilometers and a ration between the propagation velocities of 1.75 , these results characterize the continental crust, making an estimation of how the converted waves due the Mohorovičić discontinuity that arrive and interact between each other, to collect the pertinent information of the medium under study, applying a model that is based on give different values to weight factors for each wave that arrived considering geological and statistical factors.

Keywords: Crust, Mohorovičić, Function, Receiver, TGUH

En este trabajo se presenta el estudio realizado de todos los eventos que han sido registrados por la estación sismológica TGUH ubicada en Tegucigalpa, Honduras desde septiembre de 2006 hasta julio de 2014. Dichos eventos fueron seleccionados bajo ciertos criterios y características especificas que debian cumplir para poder determinar cual es el espesor de la corteza continental bajo la estación que registro los eventos, asi como la relación que existe entre las velocidades de propagación $V_{p} / V_{s}$, por medio del método de análisis de la Función Receptora que junto con el Método de Conjuntos $(H-\kappa)$ se obtuvo un modelo estimado de los parámetros de Bulk, un espesor de 34.8 kilometros y una razón entre las velocidades de propagacción de 1.75, estos caracterizan a la corteza continental, haciendo un estimado de como las ondas convertidas por la discontinuidad de Mohorovičić que arriban e interactúan entre sí para poder recolectar la información pertinente del medio que se estudio, aplicando un modelo que se basa en dar valoración con factores de peso a cada onda que arribo con base en diferentes factores geológicos y estadísticos.

Keywords: Corteza, Mohorovičić, Función, Receptora, TGUH

\section{INTRODUCCIÓN}

$\mathrm{E}$ L espesor promedio de la corteza continental tiene un valor estimado de treinta y cinco (35) kilometros. En zonas de orogenia la corteza continental puede llegar a tener un espesor entre sesenta (60) y setenta (70) kilometros, actualmente el espesor máximo alcanzado es de setenta y cinco (75) kilometros bajo la cordillera del Himalaya [12. Existen diferentes metodologías que pueden conducir a encontrar cual sería el espesor de la corteza continental, estos estudios incluye por ejemplo: "Tomografía del tiempo de viaje de la onda $P_{n}$ y/o $S_{n}$ " [6] [11, "Experimentos de sondeo sísmico profundo (DSS)" 2] y "Análisis de ondas $\mathrm{P}$ a S convertidas en el Moho" 8. La tomografía del tiempo de viaje provee una 
imagen con buena resolución de heterogeneidad entre la corteza y el manto, aunque este método baja resolución para la profundidad relativa de la discontinuidad de Mohorovičić usualmente su incertidumbre anda en el orden de diez (10) kilometros a diferencia del sondeo sísmico profundo que ofrece una mejor resolución de la profundidad de la estructura de la corteza pero su costo de adquisición es muy alto, por lo que se selecciono el método de la Función Receptora junto con el Método de Conjuntos por su confiabilidad en la resolución del espesor de la corteza continental así como su fácil adquisición y manejo de los datos.

\section{Honduras y la Función Receptora}

Actualmente Honduras no posee un modelo definitivo acerca de cual es el valor estimado del espesor de la corteza continental bajo su territorio, esto debido a la poca investigación que se realiza. El tema de la Función Receptora surge como propuesta debido a que ha sido un método que ha mostrado buenos resultados en diferentes países en todo el mundo, donde los modelos resultantes han sido de gran ayuda en el desarrollo y aplicación de nuevas metodologías. IRIS (Incorporated Research Institute for Seismology) como institución colaboradora de muchos países, ha desarrollado metodologías automatizadas para determinar el espesor de la corteza continental, mostrando resultados validos pero al ser automatizados no existe manera alguna de corroborar que estos hallan sido manejados con cuidado y analizados correctamente, por lo que se consideró un estudio mas detallado de ciertos factores de peso que se consideran en está metodología y como estos se relacionan con la geología de Honduras.

En las siguientes secciones se presentara como fue el proceso de selección de los eventos así como la definición y metodología que se debe seguir para lograr obtener la Función Receptora y como está se maneja dentro del Método de Conjuntos para estimar los parámetros de Bulk.

\section{MetodologíA}

\section{Datos de TGUH}

Desde el año 2006 la estación sismológica TGUH comenzó a funcionar (20 de septiembre de 2006) gracias a la cooperación de USGS (United States Geological Survey), la estación cuenta con diferentes instrumentos que miden los parámetros de respuesta ante eventos sismológicos en diferentes canales con la misma frecuencia de muestreo. Ubicada a una latitud de 14.0570, longitud -87.2730 y elevación de 1151 metros sobre el nivel del mar, la estación se localiza en la base militar del municipio de Tegucigalpa en Francisco Morazán.

Los registros de la estación son manejados por IRIS (Incorporated Research Institutions for Seismology) quien permite el libre acceso de todos los registros de diferentes estaciones alrededor del mundo, para su uso académico 4].

\section{Eventos Característicos}

Los requerimientos que deben cumplir los eventos para ser utilizados en el análisis de la Función Receptora y ser caracterizados como eventos ideales para este estudio son:

- Haber sucedido a una distancia de la estación entre $30^{\circ}$ y $100^{\circ}$ (ver Figura 1).

- Tener una magnitud mayor a 6.0 en la escala Ritcher y de grado VI o mayor en la escala Mercalli.

Las dos anteriores se consideran como requerimientos básicos, debido a que se espera que la onda arribe de tal forma que la onda $\mathrm{P}$ sea casi paralela a la dirección de la componente vertical del sismómetro, para ello se toma en cuenta la primera característica. La consideración de la segunda característica se basa en el recorrido de la onda, ya que recorrerá una gran distancia en la cual puede perder amplitud y al arribar está señal puede carecer de claridad entre todo el ruido y la respuesta instrumental.

\section{Función Receptora}

En 1977 Charles Langston propone el artículo Structure under Mount Rainier el cual con- 


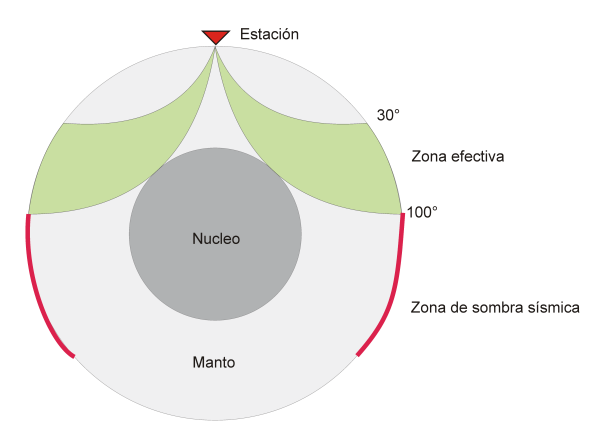

Figura 1: Zona efectiva en donde debe ocurrir el evento para ser seleccionado $\left(30^{\circ}\right.$ - 100 ), así como la zona de sombra sísmica $\left(103^{\circ}-142^{\circ}\right)$ en donde cualquier evento que ocurra aquí no será detectado por la estación.

tenía una nueva teória metodológica para poder determinar un modelo que estime el espesor de la corteza continental tomando en cuenta la información que recolecta la señal producida por un evento de gran magnitud que ocurrió a una determinada distancia, durante su recorrido por todos los sistemas que constituyen la estructura de la Tierra hasta llegar a la estación que registra la respuesta final, el considero que cuando la señal interactúa con los medios (el manto y la corteza continental) y al momento de cruzar por la discontinuidad de Mohorovičić produce ciertas transformaciones en sus ondas, como ser el cambio de $P$ a $S$ y de $S$ a $P$ y todas las repeticiones de estás. Las nuevas ondas producidas tendrán un recorrido similar aunque sus arribos serán en tiempos diferentes debido a su onda progenitora, esta diferencia fue la base de su estudio que dio como resultado la Función Receptora, en donde se maneja la interacción entre estas ondas y como se muestran y comprenden en el registro de los eventos en el receptor o estación sismológica. Por lo tanto una Función Receptora es una serie o función de tiempo que muestra la respuesta de la señal generada por un evento al interactuar con la estructura de la Tierra cerca del receptor. Está función es el resultado de la deconvolución del análisis espectral entre las componentes regis- tradas de un evento. El análisis de la Función Receptora es una forma robusta y elegante de aislar el efecto de la fuente de la respuesta de la estructura de la Tierra, que usualmente esta compuesta por una serie de ondas transformadas $\mathrm{P}$ a $\mathrm{S}$ y viceversa, que se repiten y resuenan debajo de la estación (sismómetro).

De acuerdo a Langston (1977) [5] quien fue el impulsor del tema y metodología conocida como Función Receptora, se considera el registro del evento como el desarrollo en convolución de las respuestas de cada sistema lineal hasta llegar a ser el registro del sismómetro o respuesta final y se expreso de la siguiente forma:

$$
D_{j}(t)=I(t) * S(t) * E_{j}(t)
$$

En donde el termino $D_{j}(t)$ seria el registro de cada componente del sismómetro (ZVertical, N-Norte, E-Este), $I(t)$ es la respuesta instrumental del sistema de registro, $S(t)$ es el efecto de la fuente sísmica y del manto profundo y $E_{j}(t)$ es el efecto de la corteza continental y del manto superior (su contacto seria la discontinuidad de Mohorovičić), todas ellas en el dominio del tiempo $t$ y considerando que la respuesta instrumental así como el efecto de la fuente sísmica y del manto profundo serán las mismas para cada componente del registro del evento.

\section{Sistemas de Coordenadas}

Antes de ser utilizados estos registros deben ser proyectados del sistema de coordenadas ZNE (Vertical-Norte-Este) al sistema ZRT (Vertical-Radial-Transversal) el cual consiste en aplicar una transformación a cada vector del registro de las componentes con un tensor de rotación (ver Ecuación 2) que se basa en el ángulo esférico relativo entre ambas posiciones conocido como Azimuth $\left(A_{z}\right)$, el cual se mide referente a la latitud y longitud de la posición en donde ocurrió el evento y la posición de la estación (ver Figura 2).

$$
\left[\begin{array}{c}
D_{Z} \\
D_{R} \\
D_{T}
\end{array}\right]=\left[\begin{array}{ccc}
1 & 0 & 0 \\
0 & \operatorname{Cos}\left(A_{z}\right) & \operatorname{Sin}\left(A_{z}\right) \\
0 & -\operatorname{Sin}\left(A_{z}\right) & \operatorname{Cos}\left(A_{z}\right)
\end{array}\right]\left[\begin{array}{c}
D_{Z} \\
D_{N} \\
D_{E}
\end{array}\right]
$$


La transformación considera como eje principal la dirección de la vertical en donde se espera que se observe el arribo de la onda $\mathrm{P}$ con mayor claridad (considerando siempre que está esta proyectada debido al ángulo de incidencia de la onda), y proyectando así la onda $\mathrm{S}$ dentro de los otras dos componentes.

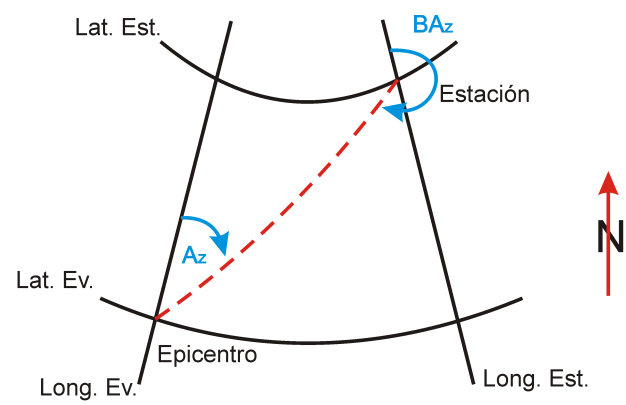

Figura 2: Ángulo azimuthal medido desde el Norte entre la posición del evento y la estación considerando su posición relativa sobre un geoide.
En muchos de los casos el efecto de la transformada no altera el resultado de los arribos de las ondas, esto es posible cuando el arribo de la onda $\mathrm{P}$ no posee un ángulo de incidencia $(\Theta)$ grande, por lo que la señal es nítida y posee valores de correlación altos entre sus componentes radial y transversal (ver Figura 3 .

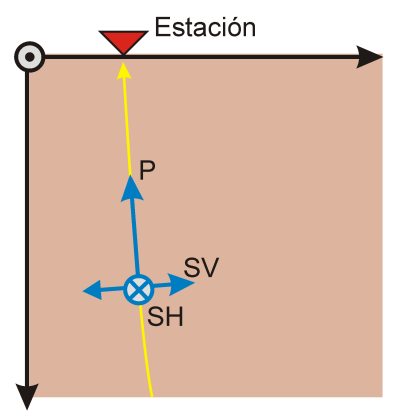

Figura 3: Arribo de la onda $P, S V$ y $S H$ a la estación con un ángulo de incidencia pequeño - caso ideal.

$$
\left[\begin{array}{c}
D_{L} \\
D_{Q} \\
D_{T}
\end{array}\right]=\left[\begin{array}{ccc}
\operatorname{Cos}(\Theta) & -\operatorname{Sin}(\Theta) \operatorname{Sin}\left(B A_{z}\right) & -\operatorname{Sin}(\Theta) \operatorname{Cos}\left(B A_{z}\right) \\
\operatorname{Sin}(\Theta) & \operatorname{Cos}(\Theta) \operatorname{Sin}\left(B A_{z}\right) & \operatorname{Cos}(\Theta) \operatorname{Cos}\left(B A_{z}\right) \\
0 & -\operatorname{Cos}\left(B A_{z}\right) & \operatorname{Sin}\left(B A_{z}\right)
\end{array}\right]\left[\begin{array}{c}
D_{Z} \\
D_{E} \\
D_{N}
\end{array}\right]
$$

Considerando el caso cuando el ángulo de incidencia $(\Theta)$ es grande se propone el uso de otro sistema de coordenadas conocido como LQT (L-Vertical o P, Q-Radial o SV y T-Transversal o $\mathrm{SH}$ ) que fue propuesto por Vinnik en 1977 [13], en este sistema se proyectan las tres componentes del registro en base a dos ángulos, el primero es el ángulo de incidencia $(\Theta)$ de la onda $\mathrm{P}$ medido desde la componente vertical, utiliza esté sistema como método para aislar los arribos de manera que se logren identificar con claridad los tres tipos de onda $\mathrm{P}, \mathrm{SV}$ y $\mathrm{SH}$, ya que proyectan todas las componentes en un sistema coordenado que toma como eje principal la dirección en que arribo la onda $\mathrm{P}$, separando el acoplamiento de estas ondas, el segundo ángulo es el Back-Azimuth $\left(B A_{z}\right)$ (ver Figura 2 el cual se referencia desde la estación hasta la posición en donde ocurre el evento, como resultado se tiene un tensor que define este nuevo sistema relativo a estos ángulos (ver Ecuación 3).

La propuesta de la Función Receptora nos dice que por medio del análisis de Fourier se puede aislar el efecto de la corteza continental y del manto superior para poder analizarlo y determinar los parámetros de Bulk que caracterizan la corteza, para lograr esto se utilizan métodos de deconvolución iterativa que permiten generar la Función Receptora desde los registros ya proyectados en cualquiera de los sistemas coordenados. Ciertas consideraciones a tomar en cuenta son:

- La corteza continental se tomara como un solo estrato horizontal.

- La respuesta del efecto de la corteza continental en dirección vertical se considera como $E_{Z}(t)=\delta(t)$.

Estas consideraciones se toman en cuenta basados en que el desarrollo de la Función Re- 
ceptora es para determinar el espesor de la corteza en un punto en especifico, por lo que el considerar la misma como un estrato horizontal es pertinente, La segunda consideración corresponde al arribo de la onda $\mathrm{P}$, ya que se espera que está llegue lo más paralela posible a la componente vertical (se tomaría como un solo un impulso). El efecto de la corteza continental y del manto superior ya aislado en el dominio de la frecuencia sería:

$$
E_{i}(\omega)=\frac{D_{i}(\omega)}{D_{Z}(\omega)}
$$

El subíndice $i$ especifica que puede ser tanto para la componente radial como para la transversal. Dichas señales tienen mucho ruido en ciertas ocasiones y este perjudica el análisis resultando por lo que se procede a normalizar la función respecto al registro de la componente vertical, este paso mejora la señal pero muestra valores erróneos al encontrar magnitudes cercanas a cero por lo que Dey-Sarkar y Wiggins (1976) 3] proponen un método de corrección para este fenómeno y es conocido como "Water-Level Process" (ver Ecuación 7) el cual regula en función al valor máximo de la señal transformada, llevando la señal a un valor medio relativo, así mismo se aplica una Gaussiana que suaviza la señal (ver Ecuación 6). Ambos filtros son aplicaciones que modifican la ecuación 4 y proponen la ecuación 5 .

$$
E_{i}(\omega)=\frac{D_{i}(\omega) D_{Z}^{*}(\omega) G(\omega)}{\Phi(\omega)}
$$

En donde:

$$
\begin{gathered}
G(\omega)=\exp \left(-\frac{\omega^{2}}{a^{2}}\right) \\
\Phi(\omega)=\max \left(D_{Z}(\omega) D_{Z}^{*}(\omega), c \max \left[D_{Z}(\omega) D_{Z}^{*}(\omega)\right]\right)
\end{gathered}
$$

Con $a$ y $c$ como constantes de cada filtro respectivamente mencionados, la selección de estas constantes debe basarse en la señal registrada (como está se presenta). Estas ecuaciones se encuentran en el dominio de la frecuencia $\omega$ aunque la verdadera Función Receptora deberá estar en el dominio del tiempo $t$ (ver Figura 4), diferentes rutinas han sido generadas para esta clase de procedimiento, la más utilizada en esta clase de estudios es la de Charles Ammon quien la propone en 1999 [7] una rutina de deconvolución iterativa que demostró gran aceptación con base a su funcionalidad, y luego él permitió que fuese de libre acceso para académicos.

\section{Método de Conjuntos $(\mathrm{H}-\kappa)$}

Este método consiste en apilar o juntar una serie de eventos (sus Funciones Receptoras) para poder determinar el valor efectivo de cada parámetro de Bulk ( $H$ el espesor de la corteza continental y $\kappa$ el valor de la razón entre las velocidades de propagación), los cuales describen el efecto de la Corteza Continental bajo el receptor que las registro (Zhu y Kanamori, 2000; Chevrot y van der Hilst, 2000; Niu y James, 2002; Nair et al., 2006) [14, [1, [10, [9] (ver Ecuación 8).

$$
s(H, \kappa)=\frac{1}{N} \sum_{i=1}^{N}\left(\omega_{1} E_{i}\left(t_{1}\right)+\omega_{2} E_{i}\left(t_{2}\right)-\omega_{3} E_{i}\left(t_{3}\right)\right)
$$

En donde:

- $N=$ número de funciones receptoras en una estación dada.
- $E_{i}(t)=$ representa la amplitud de la $i$ ésima función receptora.

- $t_{1}=$ tiempo de arribo de la onda $P_{s}$. 


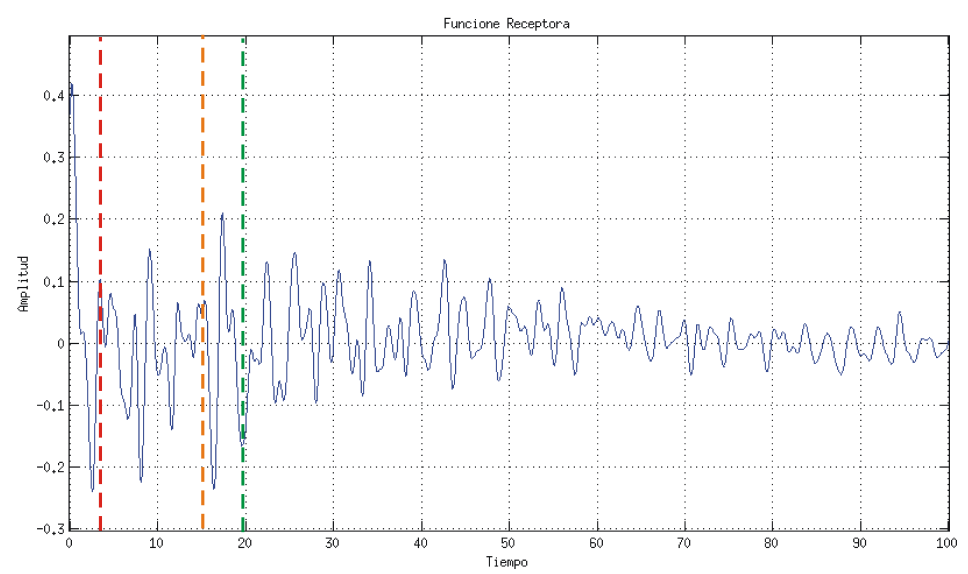

Figura 4: Función Receptora Radial generada de un evento ocurrido cerca de la costa de Chile (-38.4, -73.4) con una magnitud de 6.6 MB. La linea vertical roja marca el arribo de la onda $P_{s}$, la anaranjada el de la onda $P_{p} P_{s}$, y la verde el de la onda $P_{s} P_{s}+P_{p} S_{s}$.

- $t_{2}=$ tiempo de arribo de la onda $P_{p} P_{s}$.

- $t_{3}=$ tiempo de arribo de la onda $P_{s} P_{s}+$ $P_{p} S_{s}$.

- $\omega_{1}, \omega_{2} y \omega_{3}=$ factores de peso.

Los tiempos pueden ser estimados por medio de los parámetros de Bulk y de la velocidad promedio de propagación de la onda $P$ y su vector de lentitud $p$. El signo que acompaña a cada termino de la expresión corresponde a la polaridad de la onda que arriba, en este caso el arribo de la onda " $P_{s} P_{s}+P_{p} S_{s}$ " es negativo por lo que se le aplica el signo para invertir este valor. El conocimiento de la polaridad del arribo de cada onda es relevante debido a la relación que tienen todas ellas al realizar el método de conjuntos en donde se analiza la proporción por relevancia de cada una para determinar los parámetros efectivos para el modelo de la corteza bajo la estación (ver Ecuaciones 9, 10 y 11 .

$$
\begin{gathered}
t_{1}=H\left(\sqrt{\left(\frac{\kappa}{V_{P}}\right)^{2}-p^{2}}-\sqrt{\left(\frac{1}{V_{P}}\right)^{2}-p^{2}}\right) \\
t_{2}=H\left(\sqrt{\left(\frac{\kappa}{V_{P}}\right)^{2}-p^{2}}+\sqrt{\left(\frac{1}{V_{P}}\right)^{2}-p^{2}}\right) \\
t_{3}=2 H\left(\sqrt{\left(\frac{\kappa}{V_{P}}\right)^{2}-p^{2}}\right)
\end{gathered}
$$

El enfoque de este método es determinar la mejor relación entre ambos parámetros de Bulk de forma que estos se ajusten a la señal obtenida para cada arribo de las ondas. La Función Receptora como tal, permite determinar el arribo de cada onda a simple vista cuando la definición de la misma es lo suficientemente sensible para notarlo, en la mayoría de los casos esto no es así por lo que se procede y desarrolla la metodología mencionada en donde se toman los factores de peso $\omega$ para poder regular la relevancia de cada tipo de conversión de onda en el modelo, dichos factores se basan en diferentes consideraciones tanto geológicas como estadísticas para ser determinados, y son diferentes para cada 
estación que se este estudiando, IRIS como tal maneja dichos valores con igual relevancia $\left(\omega_{1}=1 / 3, \omega_{2}=1 / 3\right.$ y $\left.\omega_{3}=1 / 3\right)$ en donde considera una distribución pareja del 1.0 entre las tres ondas, diferentes investigadores que han trabajado el tema analizan la evolución de sus territorios y definen diferentes factores para sus estaciones, por ejemplo Zhu y Kanamori, 2000 [14] emplearon $\omega_{1}=0.7, \omega_{2}=0.2$ y $\omega_{3}=0.1$ en su estudio para determinar la profundidad del Moho en el sur de California; Chevrot y van der Hilst, 2000 [1] utilizaron $\omega_{1}=0.5, \omega_{2}=0.5$ y $\omega_{3}=0.0$ en la estimación de la razón de Poisson para la corteza Australiana y Gao et al., 2006 9] emplearon $\omega_{1}=0.5, \omega_{2}=0.3$ y $\omega_{3}=0.2 \mathrm{en} \mathrm{su}$ estudio de las restricciones de la corteza en el sur de África. La determinación de los factores de peso ideales para cada zona es de suma

De cada evento se tomo una serie de tiempo de dos minutos, este registro corresponde a un minuto antes del arribo de la onda $\mathrm{P}$ y un minuto luego del arribo de la misma, se realizo esto debido a que normalmente se espera que el arribo de las ondas $P_{s}, P_{p} P_{s}$ y $P_{s} P_{s}+P_{p} S_{s}$ se encuentren en los primeros veinte (20) segundos del evento, estos arribos serán mas notables en la Función Receptora (ver Figura 4 ).

\section{Resultados}

Los resultados del análisis de la Función Receptora se basan en la comparación de todas las funciones generadas por medio de todos los eventos seleccionados (ver. Figura 5).

El diagrama de estimación es el resultado final del análisis de la Función Receptora, por medio de este podemos observar como se da la interacción de todas las Funciones Receptoras entre sí, luego de pasar por el método de conjuntos en donde se encontraron los parámetros de Bulk efectivos para cada Función Receptora, aunque dichos valores no necesariamente serán correspondientes al resultado final, el relevancia al momento de realizar estudios por medio del análisis de la Función Receptora.

\section{Datos o Eventos SELECCIONADOS}

Se utilizaron un total de treinta y cuatro (34) eventos para generar las Funciones Receptoras para el cálculo del espesor de la corteza continental $\mathrm{H}$ y de la razón de velocidades de propagación $\kappa$ para la estación TGUH usando la metodología de conjuntos $(\mathrm{H}-\kappa)$. Los treinta y cuatro (34) eventos seleccionados no solo cumplen con las características solicitadas para el análisis de la Función Receptora, también cumplen con una correlación entre sus componentes mayor al noventa por ciento $(90 \%)$ (ver Cuadro 17.

cual dependerá de la cantidad de funciones receptoras obtenidas, en donde se observa que las compatibilidades que poseen entre ellas predominan por sobre los máximos aparentes que poseen individualmente (ver Figura 6), estos parámetros determinados en conjunto serán los resultados finales y validos a tomar, debido a que mostraran la correlación que poseen todas las funciones receptoras (ver Ecuación 8).

El primer diagrama (ver Figura 6) que se muestra es el trabajado con base a los factores de peso seleccionados por IRIS para la estación $\operatorname{TGUH}\left(\omega_{1}=1 / 3, \omega_{2}=1 / 3\right.$ y $\left.\omega_{3}=1 / 3\right)$ y en este no se removió la media de las señales registradas por el sismómetro de la estación. Así mismo se realizo el calculo del modelo estimado de los parámetros de Bulk con la excepción de que si se removió la media de la señal para este modelo (ver Figura 77). Finalmente se lograron determinar los factores adecuados que lograran aclarar cuales serían los posibles valores de los parámetros que definen el modelo de la corteza continental bajo la estación TGUH en este caso los valores resultaron ser $\omega_{1}=0.5$, $\omega_{2}=0.2$ y $\omega_{3}=0.3($ ver Figura 8 . 


\begin{tabular}{|c|c|c|c|c|c|}
\hline \# & Region & Tiempo & Lat/Lon & Depth & Distancia \\
\hline 1 & Samoa Islands Region & 2006/09/08, 06:22:09 GMT & $-16.6 /-172.0$ & $28 \mathrm{~km}$ & $89.1^{\circ}$ \\
\hline 2 & Near Coast of Central Chile & 2011/01/02, 20:20:17 GMT & $-38.4 /-73.3$ & $24 \mathrm{~km}$ & $53.7^{\circ}$ \\
\hline 3 & South of Fiji Islands & 2013/05/23, 17:19:04 GMT & $-23.0 / 177.2$ & $174 \mathrm{~km}$ & $95.3^{\circ}$ \\
\hline 4 & Northern Chile & 2007/11/14, 15:40:50 GMT & $-22.2 /-69.9$ & $40 \mathrm{~km}$ & $39.9^{\circ}$ \\
\hline 5 & Fox islands, Aleutian Islands & 2011/06/24, 03:09:39 GMT & $52.0 /-171.8$ & $52 \mathrm{~km}$ & $75.7^{\circ}$ \\
\hline 6 & Fox islands, Aleutian Islands & 2010/07/08, 05:56:44 GMT & $52.9 /-169.8$ & $14 \mathrm{~km}$ & $74.5^{\circ}$ \\
\hline 7 & Near Coast of Central Chile & 2011/02/11, 20:05:30 GMT & $-36.5 /-73.1$ & $28 \mathrm{~km}$ & $52.0^{\circ}$ \\
\hline 8 & Andreanof Islands, Aleutian Islands & 2008/05/02, 01:33:37 GMT & $51.9 /-177.5$ & $14 \mathrm{~km}$ & $79.2^{\circ}$ \\
\hline 9 & Andreanof Islands, Aleutian Islands & 2013/09/04, 02:32:30 GMT & $-37.6 /-73.7$ & $20 \mathrm{~km}$ & $77.6^{\circ}$ \\
\hline 10 & Near Coast of Central Chile & 2011/06/01, 12:55:22 GMT & $-35.2 /-72.2$ & $21 \mathrm{~km}$ & $52.9^{\circ}$ \\
\hline 11 & Near Coast of Central Chile & 2012/03/25, 22:37:06 GMT & $53.9 / 152.9$ & $41 \mathrm{~km}$ & $51.0^{\circ}$ \\
\hline 12 & Sea of Okhotsk & 2008/07/05, 02:12:04 GMT & $-60.3 /-46.4$ & $633 \mathrm{~km}$ & $95.2^{\circ}$ \\
\hline 13 & Scotia Sea & 2013/11/17, 09:04:55 GMT & $-22.2 /-65.8$ & $10 \mathrm{~km}$ & $81.0^{\circ}$ \\
\hline 14 & Jujuy Province, Argentina & 2007/07/21, 15:34:52 GMT & $51.8 / 178.8$ & $290 \mathrm{~km}$ & $41.7^{\circ}$ \\
\hline 15 & Rat Islands, Aleutian Islands & 2014/06/23, 20:53:09 GMT & $51.2 / 178.8$ & $108 \mathrm{~km}$ & $81.5^{\circ}$ \\
\hline 16 & Rat Islands, Aleutian Islands & 2008/05/20, 13:53:35 GMT & $-55.5 /-28.5$ & $27 \mathrm{~km}$ & $81.7^{\circ}$ \\
\hline 17 & South Sandwich Islands Region & 2014/06/29, 07:52:56 GMT & $51.9 /-179.4$ & $16 \mathrm{~km}$ & $85.0^{\circ}$ \\
\hline 18 & Andreanof Islands, Aleutian Islands & 2008/04/15, 22:59:51 GMT & $7.3 /-34.9$ & $10 \mathrm{~km}$ & $80.4^{o}$ \\
\hline 19 & Central Mid-Atlantic Ridge & 2008/05/23, 19:35:34 GMT & $52.8 /-132.1$ & $9 \mathrm{~km}$ & $51.8^{\circ}$ \\
\hline 20 & Queen Charlotte Islands Region & 2012/10/28, 03:04:08 GMT & $51.4 /-178.6$ & $14 \mathrm{~km}$ & $52.4^{\circ}$ \\
\hline 21 & Andreanof Islands, Aleutian Islands & 2010/08/04, 12:58:24 GMT & $53.1 / 171.2$ & $27 \mathrm{~km}$ & $80.0^{\circ}$ \\
\hline 22 & Near Islands, Aleutian Islands & 2010/12/23, 14:00:32 GMT & $-38.1 /-73.3$ & 18 km & $85.7^{\circ}$ \\
\hline 23 & Near Coast of Central Chile & 2010/07/14, 08:32:21 GMT & $51.5 /-175.2$ & $22 \mathrm{~km}$ & $53.5^{\circ}$ \\
\hline 24 & Andreanof Islands, Aleutian Islands & 2013/08/30, 16:25:02 GMT & $-29.0 /-176.2$ & $29 \mathrm{~km}$ & $77.9^{\circ}$ \\
\hline 25 & Kermadec Islands region & 2011/10/21, 17:57:16 GMT & $51.9 /-179.2$ & $33 \mathrm{~km}$ & $95.8^{\circ}$ \\
\hline 26 & Andreanof Islands, Aleutian Islands & 2008/04/16, 05:54:16 GMT & $-53.9 /-55.0$ & $13 \mathrm{~km}$ & $80.2^{\circ}$ \\
\hline 27 & Falkland Islands Region & 2013/11/25, 06:27:33 GMT & $51.4 /-175.4$ & $12 \mathrm{~km}$ & $73.1^{\circ}$ \\
\hline 28 & Andreanof Islands, Aleutian Islands & 2010/10/08, 03:26:13 GMT & $54.7 / 162.3$ & $19 \mathrm{~km}$ & $78.0^{\circ}$ \\
\hline 29 & Near Coast of Kamchatka & 2013/11/12, 07:03:51 GMT & $40.8 /-125.1$ & 43 km & $90.0^{\circ}$ \\
\hline 30 & Off Coast of Northern California & 2014/03/10, 05:18:13 GMT & $-29.1 /-71.2$ & 17 km & $42.4^{\circ}$ \\
\hline 31 & Near Coast of Central Chile & 2012/11/14, 19:02:06 GMT & $-61.0 /-56.1$ & $63 \mathrm{~km}$ & $45.6^{\circ}$ \\
\hline 32 & South Shetland Islands & 2012/01/15, 13:40:16 GMT & $-29.5 /-176.3$ & $10 \mathrm{~km}$ & $78.8^{\circ}$ \\
\hline 33 & Kermadec Islands Region & 2011/07/06, 19:03:18 GMT & $15.2 /-45.2$ & $17 \mathrm{~km}$ & $96.0^{\circ}$ \\
\hline 34 & Nothern Mid-Atlantic Ridge & 2013/09/05, 04:01:36 GMT & $53.1 / 173.0$ & 10 km & $40.7^{\circ}$ \\
\hline
\end{tabular}

Cuadro 1: Eventos seleccionados para el análisis de la Función Receptora por medio del Método de Conjuntos. Todos los eventos de seleccionados cumplen con las características necesarias con un porcentaje de coincidencia radial mayor a noventa por ciento (90\%)

\section{Discusión}

Durante el proceso de filtrado y limpieza de la señal se debe tomar en cuenta que está debe ser regulada para obtener una magnitud similar para los tres canales de registro, por lo que se procede a remover la media de cada componente teniendo así solo las variaciones del conteo, esto es apreciable al comparar la figura 6 con la figura 7 en donde la única diferencia entre ambas es el paso que se menciona anteriormente, observando de esta manera que este paso es primordial para el análisis de la Función Receptora ya que el resultado final obtenido por medio del Método de Conjuntos muestra que al remover la media se obtuvo mayor claridad en el modelo estimado disminu- yendo la cantidad de máximos falsos, generados por las difentes magnitudes entre los registros.

$\mathrm{Al}$ analizar los resultados se observar que no todas las Funciones Receptoras presentaron el mismo comportamiento luego de ser calculadas, se considera que el uso de los mismas constantes para los filtros aplicados a ellos no permitió que estas presentasen el resultado esperado, al compararlas en conjunto (ver Figura 5). En el caso ideal se espera que que el arribo de las ondas convertidas sea claro a diferencia de lo que se logra apreciar en la imagen, no todas las ondas tienen coincidencias entre sí. Normalmente se espera que los tiempos de arribo para las ondas se encuentren por debajo de 


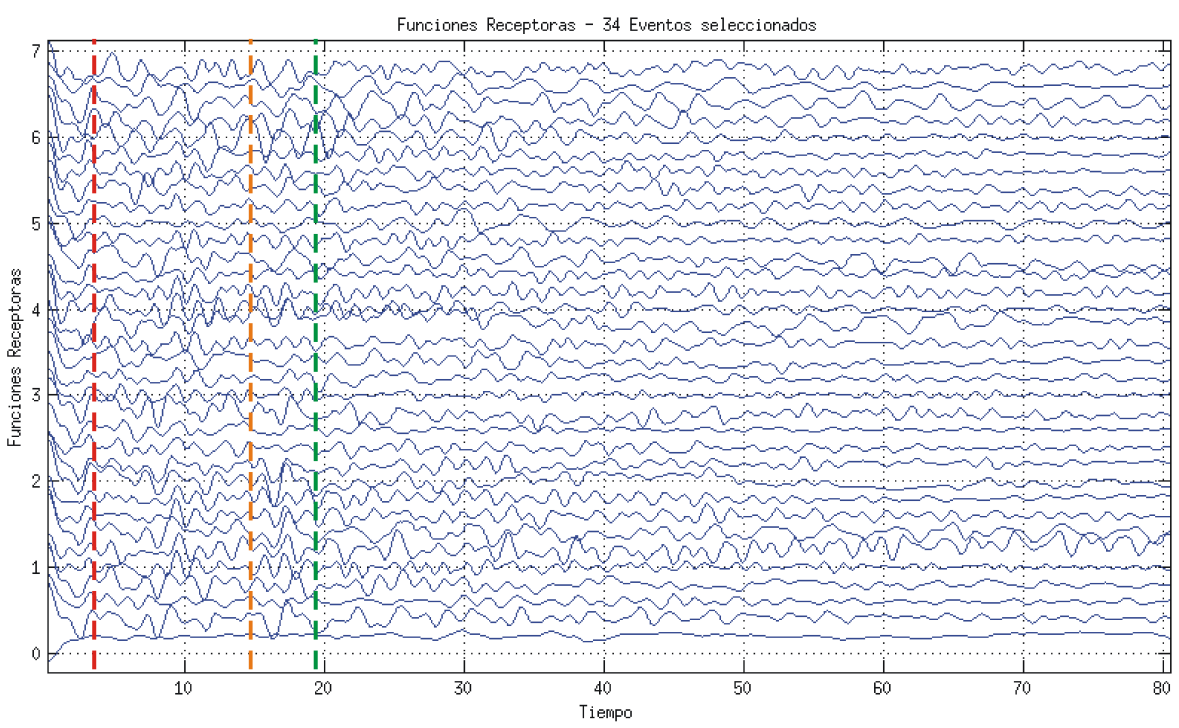

Figura 5: Funciones Receptoras calculados de los treinta y cuatro (34) eventos seleccionados. La linea vertical roja marca el arribo de la onda $P_{s}$, la anaranjada el de la onda $P_{p} P_{s}$, y la verde el de la onda $P_{s} P_{s}+P_{p} S_{s}$

los veinte (20) segundos de registro (apreciable en la Función Receptora, ver Figura 4 ya que como finalidad del Método de Conjuntos se tiene es encontrar los valores de los parámetros de Bulk que generen los tiempos correctos para los arribos de las ondas (ver Ecuaciones 9,10 y 11 así con estos tiempos se lograría determinar las amplitudes de los arribos que formen el valor máximo del conjunto siendo este sería el punto clave del modelo, en donde se tienen los parámetros que lo definen y caracterizan.

La determinación de estos tiempos es un paso clave dentro del análisis de la Función Receptora por medio del Método de Conjuntos ya que no solo depende de los valores correctos de los parámetros de Bulk, sino que también depende de los factores de peso para cada onda convertida que arriba, para determinar estos factores se baso en la claridad de los arribos así como la amplitud de los mismos presentes en cada señal. Los factores de peso manejados fueron $\omega_{1}=0.5, \omega_{2}=0.2$ y $\omega_{3}=0.3$ los cuales se seleccionaron basados en las siguientes observaciones: Se considero la amplitud de cada arribo tomando en cuenta un intervalo posible de tiempo en el cual se podría encontrar cada onda convertida, lo que llevo a apreciar que para la onda $P_{s}$ se tenia un valor medio en comparación a los demás arribos y tomando en cuenta que es la primer conversión que arriba, se selecciono un valor de 0.5 para la proporción de esta en el Método de Conjuntos, al analizar la onda $P_{p} P_{s}$ se observa que en el intervalo en el que esta debe de aparecer la mayoría de los arribos en todas las funciones receptoras presenta una superposición de ondas en la mayoría de los arribos, se considero que debido a la presencia de esto, el factor que debía poseer la proporción de la amplitud de esta onda debería de ser bajo por lo que se le asigno 0.2 , respecto a la onda $P_{s} P_{s}+P_{p} S_{s}$ su arribo fue más claro que las dos anteriores pero su amplitud tenia un valor considerablemente alto en muchas de las funciones receptoras, por lo que considero regular el mismo con una proporción de 0.3 .

Analizando el diagrama obtenido luego de aplicar los factores de peso (ver Figura 8) se encontró dentro del modelo estimado una menor cantidad de máximos, ahora dentro de estos se encuentra que el máximo de acuerdo al modelo debe de ser el punto en donde los parámetros de Bulk son $H=32.4$ kilometros y $\kappa=1.59$, pero al considerar estos valores se nota que solo el valor para el espesor de la corteza continental se encuentra cercano al valor promedio 


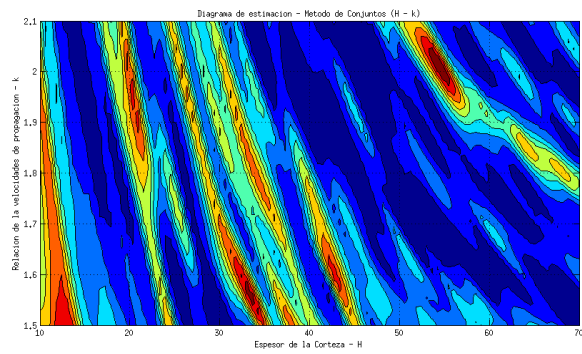

Figura 6: Diagrama de estimación sin haber removido la media de la señal y aplicando los factores de peso que maneja IRIS para la estación $T G U H$.

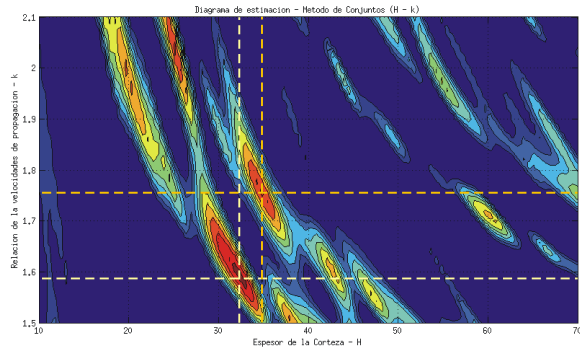

Figura 8: Diagrama de estimación calculado con factores que determinen los posibles valores de los parámetros que definen el modelo de la corteza continental bajo TGUH. Ambos cruces de las lineas punteadas marcan las dos posibles respuestas.

que se espera obtener de acuerdo a estudios anteriores de la corteza continental en América, a diferencia del valor de la razón entre las velocidades de propagación el cual se encuentra muy por debajo del valor comparable que se obtiene al analizar eventos sísmicos por medio del Método de Wadati se encontró que el valor esta alrededor de 1.77, tomando en cuenta esto se considera que el verdadero valor de la estimación seria el segundo máximo que se encuentra de acuerdo a los siguientes valores de los parámetros de Bulk $H=34.8$ kilometros y $\kappa=1.75$. Al comparar este resultado con el de IRIS es apreciable la diferencia por distintas razones, como ser los diferentes valores de los factores de peso aplicados en el Método de Conjuntos, así como la cantidad de eventos empleados en donde IRIS en su momento utilizo 133 eventos todos con un porcentaje de coincidencia mayor al ochenta por ciento $(80 \%)$ a diferencia de los 34 eventos que se

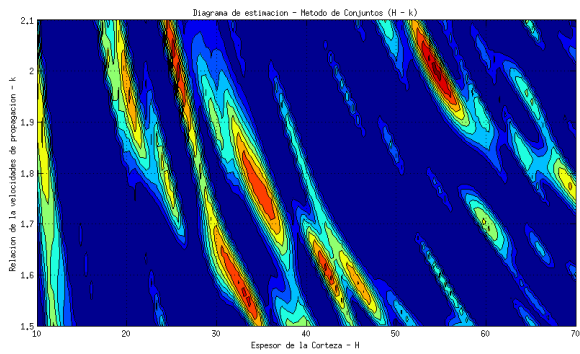

Figura 7: Diagrama de estimación habiendo removido la media de la señal y aplicando los factores de peso que maneja IRIS para la estación TGUH.

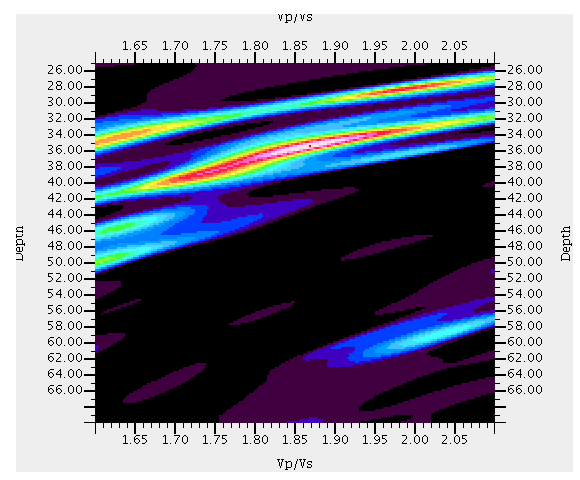

Figura 9: Diagrama de estimación calculado por IRIS para el modelo de la corteza continental bajo TGUH.

seleccionaron con un porcentaje de coincidencia mayor al noventa por ciento $(90 \%)$, dichos eventos son los que junto a los factores de peso pueden crear tal discrepancia.

Este modelo puede ser mejorado al analizar el hecho de que no todas las Funciones Receptoras poseen la misma relevancia, debido a que estas son generadas por las señales producidas por los eventos loa cuales se pueden ver afectados por su recorrido a través de las estructuras que encuentran en su camino, considerando que se pueden tener perdidas y modificaciones. Por lo que se propone hacer un análisis de factibilidad para las Funciones Receptoras calculados en donde se determine un factor de peso para cada una de ellas, tomando en cuenta su localización dentro del globo terráqueo, así como la claridad y correlación de sus registros. 


\section{CONCLUSIONES}

El método de conjuntos demostró ser una metodología adecuada para determinar el espesor de la corteza continental así como la razón de las velocidades de propagación, considerando que la metodología debido a su forma simple como expresión queda abierta a modificaciones y ajuste basados en sus componentes primordiales. Como finalidad se puede concluir que el valor posible que se puede esperar para el espesor de la corteza continental es de 34.8 kilometros, así mismo se concluye que la razón entre las velocidades de propagación es de 1.75 .

\section{REFERENCIAS}

[1] S. Chevrot and R. D. van der Hilst. The poisson ratio of the australian crust: Geological and geophysical implications. Earth Planet Sci. Lett., 183:121-132, 2000.

[2] Q. Li C. Wu Z. Yin Cui, Z. and H. Liu. The crustal and deep structures in golmudejin qi ggt. Acta Geophys. Sin., 38, 1995.

[3] S. K. Dey-Sarkar and R. A. Wiggins. Upper mantle structure in western canada. J. Geophys. Res., 81:3619-3632, 1976.

[4] IRIS. Station summary, 2012.

[5] C. A. Langston. The effect of planar dipping structure on source and receiver responses for constant ray parameter. Bull. Seis. Soc. Am., 67:1029-1050, 1977.

[6] Song X. Liang, C. and J. Huang. Tomographic inversion of pn travel times in china. J. Geophys. Res., 109, 2004.
[7] J. P. Ligorría and C. J. Ammon. Iterative deconvolution and receiver-function estimation. Bull. Seis. Soc. Am., 89:13951400, 1999.

[8] K. Priestley Mangino, S. and J. Ebel. The receiver structure beneath the china digital seismograph network stations. Bull. Seismol. Soc. Am., 89, 1999.

[9] Gao S. S. Liu K. H. Nair, S. K. and P. G. Silver. Southern african crustal evolution and composition: Constraints from receiver function studies. J. Geophys. Res., $111,2006$.

[10] F. Niu and D. E. James. Fine structure of the lowermost crust beneath the kaapvaal craton and its implications for the crustal formation and evolution. Earth Planet Sci. Lett., 200:120-130, 2002.

[11] Xu Z. Pei, S. and S. Wang. Sn wave tomography of the uppermost mantle beneath the china continent and adjacent regions. Chin. J. Geophys., 47:278-284, 2004.

[12] E. J. Tarbuck and F. K. Lutgens. Ciencias de la Tierra. Pearson Educación S. A., Madrid, 2005.

[13] L. P. Vinnik. Detection of waves converted from $\mathrm{p}$ to sv in the mantle. Phys. Earth Planet. Inter., 15:39-45, 1977.

[14] L. Zhu and H. Kanamori. Moho depth variation in southern california from teleseismic receiver functions. J. Geophys. Res., 105:2969-2980, 2000. 